\title{
Use of sequential Heidelberg retina tomograph images to identify changes at the optic disc in ocular hypertensive patients at risk of developing glaucoma
}

\author{
D S Kamal, D F Garway-Heath, R A Hitchings, F W Fitzke
}

\begin{abstract}
Aim-To determine if global and segmental changes in optic disc parameters of sequential Heidelberg retina tomograph (HRT) images develop in individual ocular hypertensive (OHT) patients without white on white visual field defects.

Methods-Patients and normal controls were recruited from a prospective ocular hypertension treatment trial. The subject groups consisted of 21 OHT patients who had converted to early glaucoma on the basis of visual field criteria (24-2 program on the Humphrey perimeter), 164 OHT subjects with normal visual fields, and 21 normal controls. Sequential HRT images 16-21 months apart were obtained for each subject and segmental optic disc parameters were measured to determine if any change had occurred. From the analysis of sequential HRT images of the 21 normal eyes we established normal limits of interimage variation. Individual discs in each group showing changes above the $95 \%$ limit of normal variability were then sought.
\end{abstract}

Results-Several segmental and global optic disc parameters were found to show significant change in the converter group before confirmed visual field change, confirming our previously published results. Individual optic disc analysis using the $95 \%$ limit of normal variability data demonstrated glaucomatous change in 13 out of 21 converter eyes. 47 of the 164 OHT eyes with normal visual fields showed change in global and segmental parameters in a "glaucomatous" direction above the level expected for normal variability. The parameters which changed most frequently in the OHT eyes were: global cup volume $(6.7 \%$ of discs), inferonasal cup volume $(11 \%)$, inferotemporal cup volume $(8.5 \%)$, and superotemporal cup area $(7.3 \%)$.

Conclusions-We have identified change in a subset of ocular hypertensive patients which could predate the development of glaucomatous visual field loss. The HRT could be of value in the sequential follow up of those suspected of having glaucoma by identifying eyes at risk of developing glaucoma. However, further refinement of the technique is required to eliminate some of the inherent variability of the analysis method described, and to in- crease the ability to detect at risk individuals.

(Br f Ophthalmol 2000;84:993-998)

It has been well established that clinically detectable glaucomatous change in the optic disc may precede the onset of white on white visual field defects by several years. ${ }^{1-6}$ Histological evidence of extensive loss of optic nerve fibres before detectable visual field loss supports this observation. ${ }^{78}$ If detection of glaucoma is made at an earlier stage of the disease, the institution of appropriate treatment may prevent or delay the onset of functional loss.

Reliance on visual field data to help in the diagnosis of early glaucoma poses problems, even with the automated techniques that are currently available. Variability in the performance of automated perimetry has been well documented in both normal subjects and patients with glaucoma, with higher levels of both intratest and inter-test fluctuation seen in the latter group. ${ }^{9-11}$ Reproducible field defects are therefore necessary to confirm change. ${ }^{12}$ Frequent visual field testing may be difficult to achieve, resulting in a prolonged interval between visual field progression and its detection.

The detection of glaucomatous change by analysis of optic disc parameters may provide us with a more objective means of assessment than currently available methods of visual field analysis. ${ }^{6}$ However, the main limitation to making a diagnosis of early glaucoma on the basis of clinical appearance of the optic disc is the difficulty in determining exactly what features constitute a "normal" disc because of the high degree of variability in the general population. For the same reason it can also be difficult to identify reliably "abnormal" discs. Classic examples in both groups can be assessed with confidence, but many discs may lie in between these extremes and offer little help in reaching a diagnosis. Previous studies have shown that there is limited agreement between even experienced observers in optic disc assessment, both ophthalmoscopically and using disc photographs. ${ }^{13-15}$ The specificity and sensitivity of optic disc assessment is limited with current clinical methods ${ }^{14}$ but newer methods such as the Heidelberg retina tomograph (HRT, Heidelberg Engineering, Heidelberg, Germany) may provide us with a more objective means of assessment. The HRT has been investigated as a tool in the imaging of the optic nerve head and its method of analysis has 
Table 1 Mean (SD) time interval from HRT1 to HRT2 and mean (SD) ages of subject groups

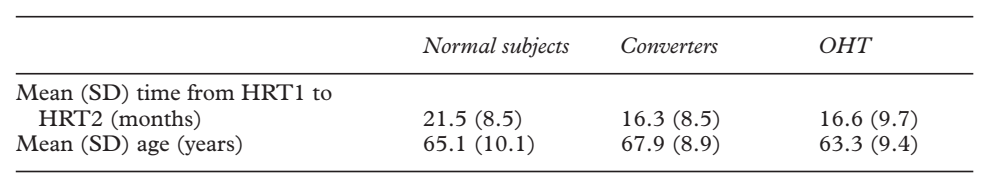

HRT $=$ Heidelberg retina tomograph; OHT $=$ ocular hypertensive.

been described elsewhere. ${ }^{16}{ }^{17}$ HRT analysis provides quantitative measures of three dimensional optic disc parameters such as cup and rim volume, as well as information on various area parameters. High levels of reproducibility have been demonstrated with the HRT. ${ }^{16}{ }^{18} 19$

The ocular hypertension (OHT) study was conducted at Moorfields Eye Hospital from 1992 to 1998 . The study included a group of 255 patients with a medium to high risk of developing ocular hypertension, a proportion of whom developed early glaucomatous field loss during the follow up period. We have called this subgroup the "converters". One of the aims of the OHT study was to evaluate several psychophysical and imaging techniques in the detection of early glaucomatous damage. All patients in the OHT study underwent serial HRT optic disc imaging from 1994 when the equipment first became available to us. A group of normal controls also underwent similar investigations in order to compare their results with those of the patients in the study.

The results reported here are a development of work previously published in $1999 .{ }^{20}$ The first part confirms our previous finding that the analysis of sequential HRT images can identify glaucomatous change in global and segmental parameters before visual field change in a larger group of converters using the latest version of HRT software. In addition, we aimed to establish the normal limits of variability of these parameters in sequential HRT imaging from a group of normal subjects and to apply these findings to a large group of OHT patients maintaining normal visual fields to determine which individuals show change in the optic disc above the normal limits.

\section{Methods}

The ethics committee at Moorfields Eye Hospital approved the OHT study and all patients and normal volunteers participating in the study gave their informed consent on initial recruitment.

All visual field testing in our study was performed using the 24-2 threshold strategy on the type 1 Humphrey field analyser. Each OHT subject underwent four monthly visual field testing.

Table 2 Optic disc parameters showing glaucomatous change in converters (group analysis by Wilcoxon signed ranks test)

\begin{tabular}{|c|c|c|c|c|c|c|c|c|}
\hline & \multicolumn{2}{|c|}{ Cup area $\left(\mathrm{mm}^{2}\right)$} & \multicolumn{2}{|c|}{ Cup volume $\left(\mathrm{mm}^{3}\right)$} & \multicolumn{2}{|c|}{ Rim area $\left(\mathrm{mm}^{2}\right)$} & \multicolumn{2}{|c|}{ Rim volume $\left(\mathrm{mm}^{3}\right)$} \\
\hline & $p$ value & $\begin{array}{l}\text { Magnitude } \\
\text { (SD) }\end{array}$ & $p$ value & $\begin{array}{l}\text { Magnitude } \\
\text { (SD) }\end{array}$ & $p$ value & $\begin{array}{l}\text { Magnitude } \\
\text { (SD) }\end{array}$ & $p$ value & $\begin{array}{l}\text { Magnitude } \\
\text { (SD) }\end{array}$ \\
\hline Global & 0.002 & $0.08(0.1)$ & 0.001 & $0.032(0.037)$ & 0.002 & $0.08(0.11)$ & $<0.001$ & $0.045(0.056)$ \\
\hline Inferotemporal & NS & $0.009(0.023)$ & 0.003 & $0.006(0.009)$ & NS & $0.009(0.023)$ & 0.001 & $0.004(0.005)$ \\
\hline Inferonasal & NS & $0.009(0.018)$ & 0.006 & $0.003(0.005)$ & NS & $0.009(0.020)$ & 0.002 & $0.007(0.010)$ \\
\hline Superotemporal & $<0.001$ & $0.023(0.030)$ & $<0.001$ & $0.008(0.007)$ & $<0.001$ & $0.023(0.03)$ & $<0.001$ & $0.006(0.007)$ \\
\hline Superonasal & $<0.001$ & $0.01(0.02)$ & NS & $0.004(0.006)$ & 0.004 & $0.012(0.020)$ & $<0.001$ & $0.007(0.009)$ \\
\hline
\end{tabular}

NS = not significant.
OHT PATIENTS

The patients were recruited from primary care and general glaucoma outpatient clinics at Moorfields Eye Hospital.

\section{Eligibility criteria}

Eligibility criteria for entry into the trial included: intraocular pressure measured by Goldmann applanation tonometry of $\geqslant 22 \mathrm{~mm} \mathrm{Hg}$ on two or more occasions; two consecutive reliable visual fields with an AGIS $^{21}$ score of 0 ; absence of any other significant ocular disease that may affect visual field performance; age more than 35 years. The clinical appearance of the optic disc as seen with a $78 \mathrm{D}$ biomicroscopic lens was not an entry criterion.

\section{Definition of conversion}

For the purposes of the OHT study we have defined conversion as follows: the change from an initial AGIS score of 0 to an AGIS score of $\geqslant 1$ on three consecutive reliable visual fields, with at least one location consistently below the threshold for normality. A reliable field was obtained when the following criteria were met: $<25 \%$ fixation losses, $<30 \%$ false negative errors, and $<30 \%$ false positive errors. The protocol in the study dictated that, if a patient developed a visual field defect on a routine four monthly visit test, this was repeated within one month and, if the same defect was then reproduced on a reliable second field, a third test was performed 3-4 months after this. Conversion was confirmed if the field defect was present on the three consecutive reliable tests. If the defect was present but the reliability indices fell outside those described above, then field defect was not reproduced on the second or third attempt, a routine perimetric appointment was given for 4 months later. Conversion was therefore confirmed by three visual field tests performed over a $4-5$ month period.

\section{NORMAL CONTROLS}

The normal controls were recruited from partners of patients, hospital staff, and other volunteers using the following eligibility criteria: intraocular pressure as measured by Goldmann tonometry of $<21 \mathrm{~mm} \mathrm{Hg}$ on two or more occasions; two consecutive visual fields with an AGIS score of 0 (acceptable reliability indices of visual field performance were the same as for the OHT group); no significant ocular disease; no family history of glaucoma or ocular hypertension; age $>35$ years. The clinical appearance of the optic disc as seen with a 78D lens was not an entry criterion. the test was repeated again within 1 month. If a 
Table $395 \%$ limit of normal variability $(1.96 \times S D$ mean difference between HRT1 and HRT2 for the normal group)

\begin{tabular}{|c|c|c|c|c|}
\hline Parameter & $\begin{array}{l}\text { Cup area } \\
\left(\mathrm{mm}^{2}\right)\end{array}$ & $\begin{array}{l}\text { Cup } \\
\text { volume } \\
\left(\mathrm{mm}^{3}\right)\end{array}$ & $\underset{\left(m m^{2}\right)}{\text { Rim area }}$ & $\begin{array}{l}\text { Rim } \\
\text { volume } \\
\left(\mathrm{mm}^{3}\right)\end{array}$ \\
\hline Global & 0.160 & 0.056 & 0.248 & 0.116 \\
\hline Inferotemporal & & 0.008 & & 0.019 \\
\hline Inferonasal & & 0.004 & & 0.017 \\
\hline Superotemporal & 0.020 & 0.013 & 0.033 & 0.014 \\
\hline Superonasal & 0.020 & & 0.036 & 0.017 \\
\hline
\end{tabular}

HRT IMAGING AND ANALYSIS

Each OHT patient included in the study had undergone annual optic disc imaging with the HRT. Normal subjects had also undergone sequential HRT imaging, but on a less frequent basis than the OHT patients. One eligible eye was included per subject.

For each HRT imaging session three $10 \times 10$ degree images were obtained for each eye by

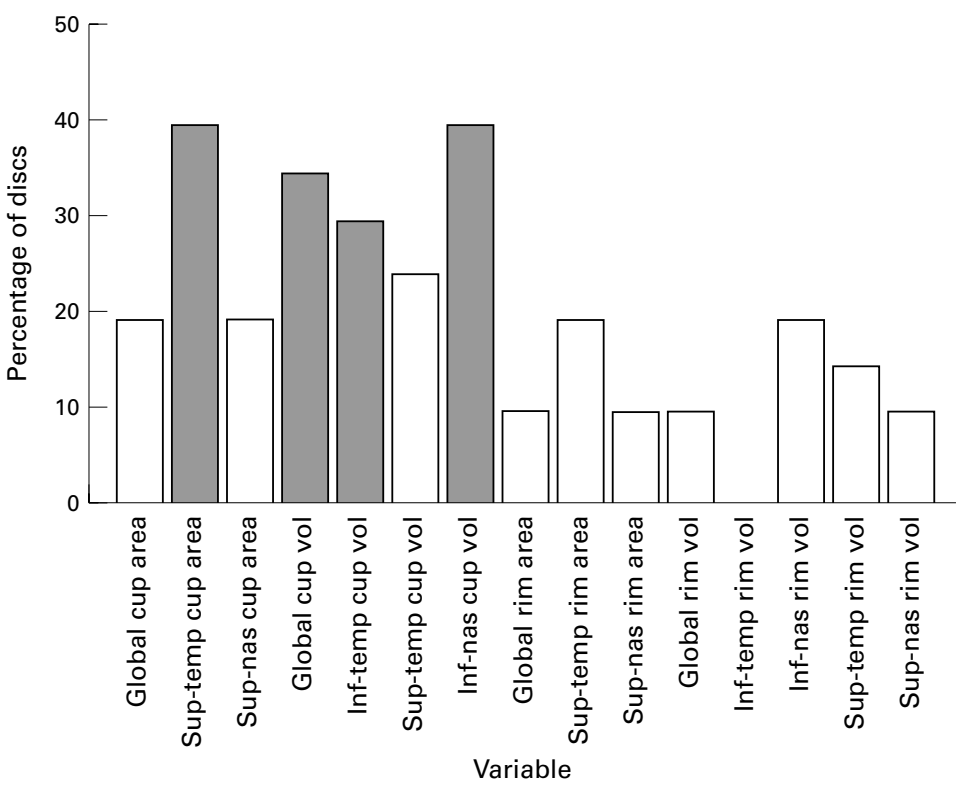

Figure 1 Parameters showing glaucomatous change in individual converter optic discs.

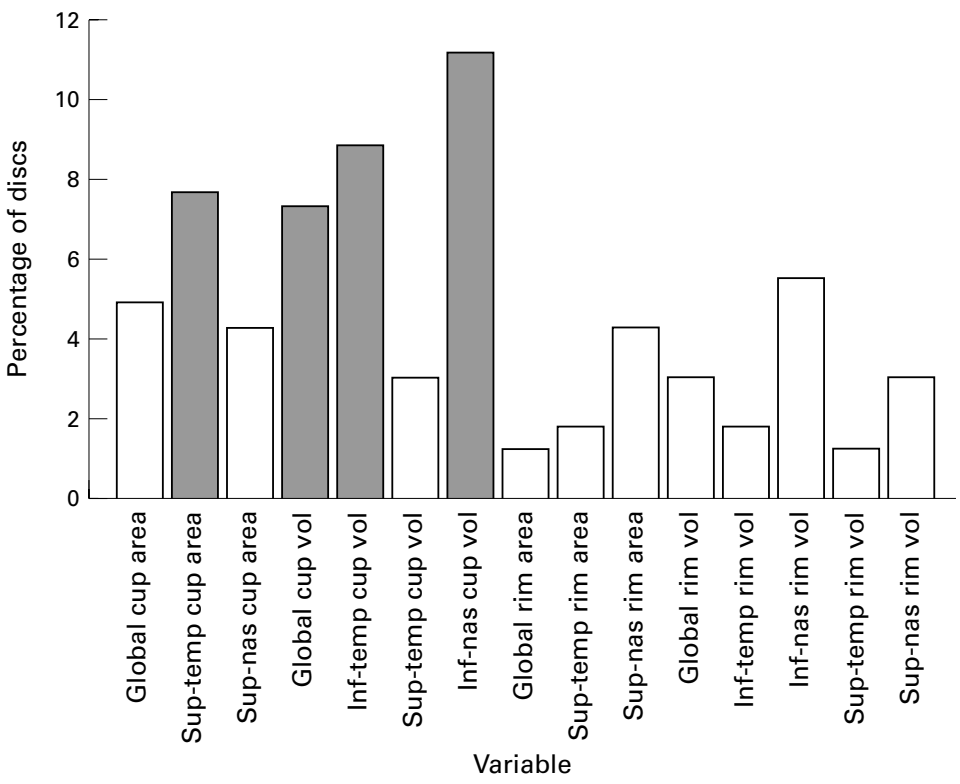

Figure 2 Parameters showing change in individual OHT optic discs. one of two experienced technicians. Images with significant movement artefact were rejected. All analysis on the HRT was performed using HRT software version 2.01. The mean topography of the three images was generated and the disc edge was delineated on the mean image by a single observer (DK) using a mouse drawn contour line. The contour line was then exported from the first HRT mean image (HRT1) onto the second HRT mean image (HRT2) of the same optic disc. In house studies have demonstrated good reproducibility levels with interobserver variability measures of neuroretinal rim values of $8.1 \%$, and intraobserver variability measures of $4.5 \% .{ }^{19}$ HRT software was used to analyse both global and segmental optic disc parameters.

The following optic disc parameters were analysed using HRT software: disc area, cup area, cup volume, rim volume and third moment (cup shape measure) using the standard reference plane.

Global parameters and six predefined optic disc segments were analysed: nasal, superonasal, inferonasal, temporal, superotemporal, and inferotemporal. For each subject included in the study two sequential HRT images were analysed. Only those subjects with good quality HRT images - that is, without any movement artefact and a mean topography standard deviation of $<20 \mu \mathrm{m}$-were included in the study analysis. The mean topographical images included in the study for each subject were directly sequential in time order.

\section{STATISTICAL METHODS}

Group analysis of changing optic disc parameters in the converters

HRT2 values were obtained before the conversion confirming third visual field (VF3). The sequential HRT image data were not distributed normally (as shown by histogram representation of the results). The non-parametric Wilcoxon signed ranks statistical test (on sPss for Windows version 7.0) was therefore used to determine if there were any statistically significant differences between the values for HRT1 and HRT2. A result was considered to be statistically significant if the $\mathrm{p}$ value was $<0.05$.

Establishing the normal limits of variability The mean magnitude of difference in parameter values between HRT1 and HRT2 for the group of normal subjects was established and the $95 \%$ limit of normal variability was determined by multiplying the standard deviation of this mean difference by 1.96 . This was calculated only for those parameters showing significant change in the converters.

Analysis of individual optic discs to identify those showing change outside the normal level of variability

All the optic discs in the three subject groups were analysed individually to determine which discs showed a magnitude of change greater than that limit established from the analysis of the normal data. Change in both directions was examined-that is, if the change was in the glaucomatous direction this was labelled as 


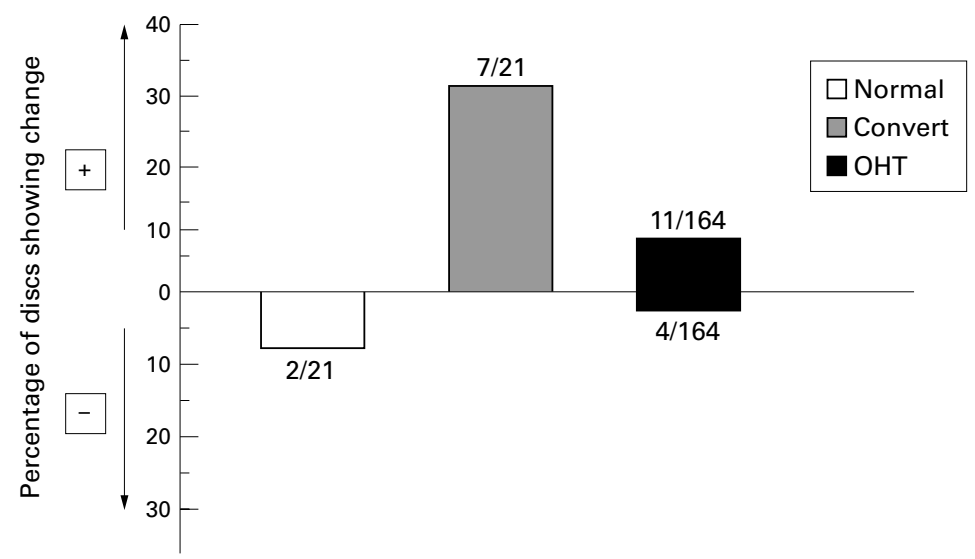

Figure 3 Direction of change in individual discs for global cup volume; + = "getting worse",-—= "getting better".

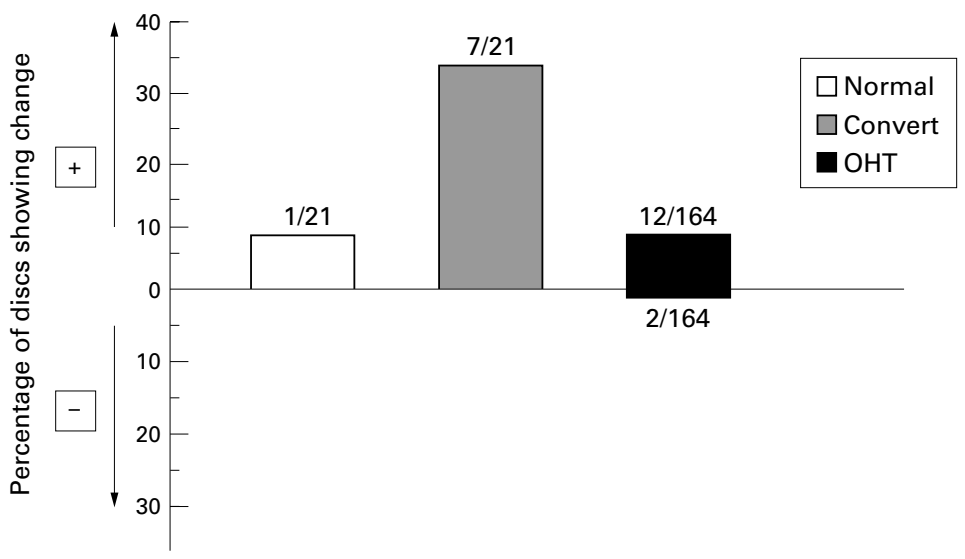

Figure 4 Direction of change in individual discs for superotemporal cup area.

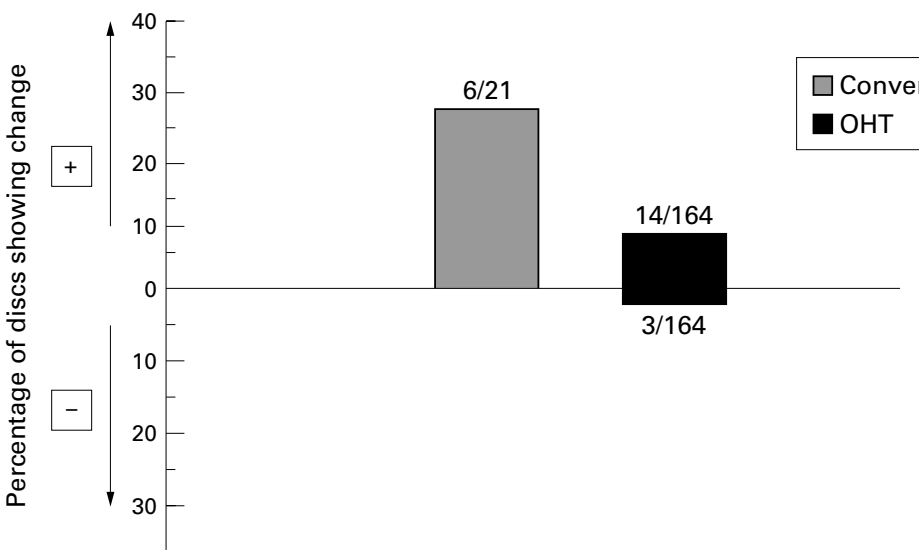

Figure 5 Direction of change in individual discs for inferotemporal cup volume.

"getting worse" represented by $(+)$ in the figures and if the change was in the opposite direction it was labelled as "getting better" represented by $(-)$ in the figures.

\section{Results}

Sequential mean topographical images from two converters, three normal subjects, and six OHT subjects were rejected because of standard deviations of more than $20 \mu \mathrm{m}$. These subjects were therefore excluded from the study.

Twenty one converters, 21 normal subjects, and 164 OHT subjects with good quality sequential HRT images were included in the study analysis. The mean ages of the groups and the time between HRT1 and HRT2 are shown in Table 1.

CONVERTER GROUP

The following segmental and global parameters were found to change ("getting worse") on the Wilcoxon analysis of the converter group data (see Table 2):

(a) Cup area: global, superonasal, and superotemporal.

(b) Cup volume: global, inferotemporal, inferonasal, superotemporal.

(c) Rim area: global, superonasal, and superotemporal.

(d) Rim volume: global, inferotemporal, inferonasal, superonasal, and superotemporal.

In all but two converters HRT2 was obtained before VF3. In two converters HRT2 was obtained at the same examination as VF3. The mean (SD) time from HRT2 to VF3 was 5.4 (5.1) months.

NORMAL GROUP

None of the parameters changed significantly in the normal group. The $95 \%$ limit of normal variability for each parameter is shown in Table 3.

INDIVIDUAL DISCS

Individual discs in the three groups of subjects were then examined to determine which ones showed change in the direction of "getting better" or "getting worse"-that is, showing change greater than the limit of normal variability.

Thirteen of the 21 converters showed change in the direction of "getting worse" and none of the converter discs showed change in the direction of "getting better"; one of the normal control discs showed change in the direction of "getting worse" and two showed change in the direction of "getting better"; 47 of the 164 OHT discs showed change in the direction of "getting worse" and 15 showed change in the direction of "getting better".

For the converters and OHT group the number of discs changing in the "getting worse" direction was plotted against each of the significant parameters shown in Table 2 . The four most frequently changing parameters were the same for both groups of subjects (Figs 1 and 2): converters (\% of 21 optic discs) global cup volume (33.3\%), inferotemporal cup volume $(28.5 \%)$, inferonasal cup volume $(38.9 \%)$, superotemporal cup area $(33.3 \%)$; OHT group (\% of 164 optic discs)-global cup volume $(6.7 \%)$, inferotemporal cup volume $(8.5 \%)$, inferonasal cup volume $(11 \%)$, superotemporal cup area $(7.3 \%)$.

These four parameters were therefore examined in more detail to identify the number of optic discs that were "getting better" as well as those "getting worse". These results are illustrated in Figs 3-6.

\section{Discussion}

A previous study published by our group ${ }^{20}$ reported on how early glaucomatous disc change was noted before the development of 


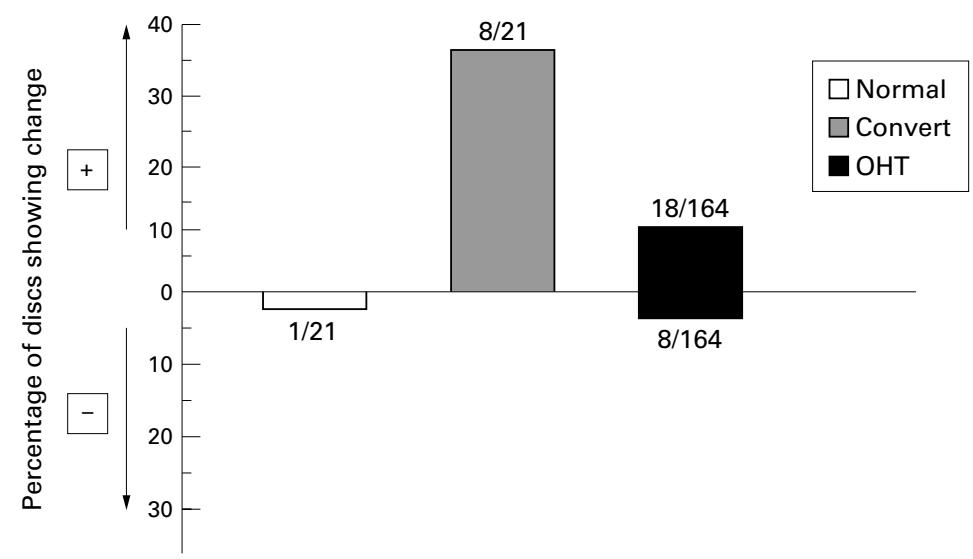

Figure 6 Direction of change in individual discs for inferonasal cup volume.

reproducible visual field defects in a group of OHT patients converting to early glaucoma. We have confirmed these results in a larger group of converters identified during the course of the OHT study at Moorfields Eye Hospital. This work looked at converter data on a group rather than on an individual basis. Following on from this we have determined the normal limit of variability of disc change using the group data obtained from a larger number of normal subjects. This methodology has enabled us to look at sequential optic disc data on an individual basis. It is apparent on individual analysis that most converter discs (13/21) do show change above that expected for normal variability but in eight cases this could not be detected by our method of HRT analysis before confirmed visual field conversion. We feel that looking for change in smaller segments may improve the method for detecting the small degrees of change associated with early glaucoma, and we will be investigating this in future work. No converter discs showed change in the direction of "getting better". Those converters who demonstrated disc change did so before visual field change.

The OHT group maintained normal visual fields but 47 individual discs were shown to be "getting worse". The $95 \%$ limit of normal variability used in the study ensures that only those OHT subjects showing significantly greater change than the normal group were identified, and we are continuing to follow these patients to determine whether they develop reproducible visual field defects. We feel, on the basis of our converter data, that these individual subjects are at risk of developing future visual field loss and they represent a group of OHT subjects who may benefit from early treatment to lower the intraocular pressure. Fifteen OHT discs also showed evidence of "getting better", which suggests that there is some inherent variability in the analysis of sequential images of HRT. Further refinement of the technique is required to reduce this variability.

Most previous studies with the HRT have been cross sectional ${ }^{22-25}$ and have concentrated on separating normal subjects from those with glaucoma. Many of these studies have been limited by the fact that they used the clinical appearance of the optic disc as an inclusion criterion for subjects. The groups have been preselected on the basis of the very parameter that is under investigation, leading to significant selection bias. ${ }^{26}$ Other studies have set out to eliminate such bias ${ }^{27-29}$ and have found relatively high levels of sensitivity and specificity of the technique in detecting glaucomatous damage to the optic disc. To our knowledge, there have not been any other published longitudinal HRT data on OHT patients with normal visual fields with which we can compare our results. At ARVO 1999 Chauhan et $a l^{\beta 0}$ presented their method for detecting changes in sequential HRT images in glaucoma patients by means of change probability maps that used data analysed on a pixel basis. Preliminary results found that this method was able to identify $95 \%$ of glaucomatous eyes with early visual field defects shown to be progressing on optic disc photography. Publication of further work using change probability maps is awaited.

In conclusion, this study has, for the first time, identified those optic discs in a group of OHT patients that show glaucomatous change over time, despite maintaining consistently normal visual fields. We believe that OHT patients who show such change are at risk of future functional visual loss and may benefit from prophylactic treatment. Our study has eliminated potential selection bias by excluding the appearance of the optic disc from the inclusion criteria for each of the subject groups. The use of the HRT in clinical practice awaits resolution of the issue of "improvement" noted in a subset of our OHT patients. Refinement of analysis methods may also resolve the issue of those converter eyes in which no change could be detected before the development of visual field defects.

Supported by The Guide Dogs For the Blind Association and the Blue Light Fund at Moorfields Eye Hospital

None of the authors has any proprietary interest in the Heidelberg retina tomograph.

1 Sommer A, Pollack I, Maumenee AE. Optic disc parameters and onset of glaucomatous field loss. I. Methods and progressive changes in disc morphology. Arch Ophthalmol 1979;97:1444-8.

2 Pederson JE, Anderson DR. The mode of progressive disc cupping in ocular hypertension and glaucoma. Arch Ophthalmol 1980;98:490-5.

3 Caprioli J. Correlation of visual function with optic nerve and nerve fibre layer structure in glaucoma. Surv Ophthalmol 1989;33:319-30.

4 Tuulonen A, Airaksinen PJ. Initial glaucomatous optic disc and retinal nerve fibre layer abnormalities and their progression. Am f Ophthalmol 1991;111:485-90.

5 Zeyen TG, Caprioli J. Progression of disc and field damage in early glaucoma. Arch Ophthalmol 1993;111:62-5.

6 Caprioli J, Prum B, Zeyen T. Comparison of methods to evaluate the optic nerve head and nerve fiber layer for glauevaluate the optic nerve head and nerve fiber layer for glau-
comatous change. Am $\mathcal{7}$ Ophthalmol 1996;121:659-67.

7 Quigley HA, Addicks EM, Green WR. Optic nerve damage Quigley HA, Addicks EM, Green WR. Optic nerve damage
in human glaucoma. III. Quantitative correlation of nerve fibre loss and visual field defect in glaucoma, ischemic neuropathy, papilledema, and toxic neuropathy. Arch Ophthalmol 1982;100:135-46.

8 Quigley HA, Kerrigan-Baumrind LA, Pease ME, et al. The number of retinal ganglion cells in glaucoma eyes compared to threshold visual field data in the same eyes. Invest Ophthalmol Vis Sci 1999;40:3059, S582.

9 Katz J, Sommer A, Witt K. Reliability of visual field results over repeated testing. Ophthalmology 1991;98:70-5.

10 Boeglin RJ, Caprioli J, Zulauf M. Long-term fluctuation of the visual field in glaucoma. Am f Ophthalmol 1992;113. 396-400.

11 Johnson CA, Nelson Quigg JM. A prospective three-year study of response properties of normal subjects and patients during automated perimetry. Ophthalmology 1993; 100:269-74.

12 Schulzer M, Anderson DR, Drance SM. Errors in the diagnosis of visual field progression in normal tension glaucoma. Ophthalmology 1994;100:99-100. 
13 Lichter PR. Variability of expert observers in evaluating the optic disc. Trans Am Ophthalmic Soc 1976;74:532-71.

14 Tielsch JM, Katz J, Quigley HA, Miller NR, et al. Intraobserver and interobserver agreement in measurement of optic disc characteristics. Ophthalmology 1988;95 $350-6$

15 Varma R, Steinmann WC, Scott IU. Expert agreement in evaluating the optic nerve head in glaucoma. Ophthalmology 1992;99:215-21

16 Cioffi GA, Robin AL, Eastman RD, et al. Confocal laser scanning ophthalmoscope. Reproducibility of optic nerve head topographic measurements with the confocal laser scanning ophthalmoscope. Ophthalmology 1993;100:5762.

17 Rohrschneider K, Burk ROW, Kruse FE, et al. Reproducibility of the optic nerve head topography with a new laser tomographic scanning device. Ophthalmology 1994;101: tomographi.

18 Chauhan BC, LeBlanc RP, McCormick TA, et al. Test-retest variability of topographic measurements with confocal scanning laser tomography in patients with
glaucoma and control subjects. Am 7 Ophthalmol 1994;118: glauco $9-15$.

19 Garway-Heath DF, Poinoosawmy D, Wollstein G, et al. Inter- and intra-observer variation in the analysis of optic disc images: a comparison of the Heidelberg retina tomograph and computer assisted tomography. $\mathrm{Br} f$ Ophthalmol 1999;83:664-9.

20 Kamal DS, Viswanathan A, Garway-Heath DF, et al. Detection of optic disc change with the Heidelberg retina tomograph before confirmed visual field change in ocular hypertensives converting to early glaucoma. $\mathrm{Br} f \mathrm{f}$ Ophthalmol 1999;83:290-4
21 AGIS Investigators. Advanced Glaucoma Intervention Study. Ophthalmology 1994;101:1445-55.

22 Zangwill LM, van Horn S, De Souza Lima M, et al. Optic nerve head topography in ocular hypertensive eyes using confocal scanning laser ophthalmoscopy. Am f Ophthalmol 1996;122:520-5.

23 Uchida H, Brigatti L, Caprioli J. Detection of structural damage from glaucoma with confocal laser image analysis. Invest Ophthalmol Vis Sci 1996;37:2393-401

24 Hatch WV, Flanagan JG, Etchells EE, et al. Laser scanning tomography of the optic nerve head in ocular hypertension and glaucoma. Br f Ophthalmol 1997;81:871-6.

25 Iester M, Mikelberg FS, Courtwright P, et al. Correlation between visual field indices and Heidelberg retina tomograph parameters. F Glaucoma 1997;6:78-82.

26 Garway-Heath DF, Hitchings RA. Quantitative evaluation of the optic nerve head in early glaucoma. Br f Ophthalmol 1998;82:352-61.

27 Mikelberg FS, Parfitt CM, Swindale N, et al. Ability of the Heidelberg retina tomograph to detect early glaucomatous field loss. F Glaucoma 1995;4:242-7.

28 Iester M, Mikelberg FS, Drance SM. The effect of optic disc size on diagnostic precision with the Heidelberg retina tomograph. Ophthalmology 1996;104:545-8.

29 Wollstein G, Garway-Heath D, Hitchings R. Identification of early glaucoma cases with the scanning laser ophthalmoscope. Ophthalmology 1997;105:1557-63.

30 Chauhan BC, McCormick TA, Leblanc R P. Utility of confocal scanning laser tomography in a long-term prospective study of patients with open-angle glaucoma. Invest Ophthalmol Vis Sci 1999;40:S397. 